\title{
Pepino mosaic virus on Tomato Seed: Virus Location and Mechanical Transmission
}

\author{
Kai-Shu Ling, U.S. Department of Agriculture-Agricultural Research Service, U.S. Vegetable Laboratory, Charles- \\ ton, SC 29414
}

\begin{abstract}
Ling, K.-S. 2008. Pepino mosaic virus on tomato seed: Virus location and mechanical transmission. Plant Dis. 92:1701-1705.

In just a few years, Pepino mosaic virus (PepMV) has become a major threat to greenhouse tomato production around the world. Although tomato seed is suspected to spread the disease, its importance as an initial virus inoculum for PepMV has not been established. To determine the potential for seed transmission, a tomato seed lot highly contaminated with PepMV was used for large-scale seedling grow-out tests. None of 10,000 grow-out seedlings was infected as determined by symptom expression, enzyme-linked immunosorbent assay (ELISA), or infectivity assay on Nicotiana benthamiana. Even though PepMV was not seed transmitted on tomato, the virus was effectively transmitted to tomato and $N$. benthamiana seedlings through mechanical transmission with seed extract. To examine the exact location where PepMV particles accumulated on the tomato seed, seed coats and embryos were carefully isolated and tested separately by ELISA, real-time RT-PCR, and bioassay on $N$. benthamiana. PepMV was detected in the seed coat fraction in both immature and mature tomato seeds, but not in the embryo. However, in $N$. benthamiana, the virus was neither seedborne nor seed-transmitted. Because PepMV is seedborne in tomato, efficient mechanical transmission of PepMV from the virus-contaminated tomato seed to seedlings could initiate a disease epidemic in a new tomato growing area. Thus, it is important to plant certified tomato seed that has been tested free of PepMV.
\end{abstract}

Additional keywords: indicator plant, potexvirus, seed health assay

Pepino mosaic is an emerging disease of tomato (Solanum lycopersicum L.) first observed in the Netherlands in 1999 (24). However, the causal agent for the disease, Pepino mosaic virus (PepMV), a member of the genus Potexvirus in the family Flexiviridae, was first described in 1980 on pepino (Solanum muricatum Aiton) in Peru (9). Despite great efforts placed on disease prevention through quarantine, cultural practice, and sanitization, the disease is still spreading rapidly in Europe $(1,4,10,13,17-21,24,25)$, North America $(6,13,14)$, South America $(11,22)$, and Asia (26). Thus, PepMV poses a great threat to an estimated $\$ 400$ million greenhouse tomato industry in the United States and much more around the world. How PepMV infection is initiated in a new area is not well understood, but tomato seed has long been suspected to be one of the key

Corresponding author: K.-S. Ling

E-mail: kai.ling@ars.usda.gov

Mention of trade names or commercial products in this article is solely for the purpose of providing specific information and does not imply recommendation or endorsement by the U.S. Department of Agriculture.

Accepted for publication 9 September 2008.

doi:10.1094/PDIS-92-12-1701

This article is in the public domain and not copyrightable. It may be freely reprinted with customary crediting of the source. The American Phytopathological Society, 2008. components responsible for the worldwide epidemic of this disease in greenhouse tomatoes. Although PepMV is easily transmitted from diseased plants to healthy ones through mechanical means, its natural transmission, if any, on the germinating tomato seedlings from PepMV-contaminated seeds is poorly understood. For example, with a small number of tomato seeds, Salomone and Roggero (21) showed no seed transmission of PepMV. In contrast, a recent publication (3) claimed to have $1.84 \%$ seed transmission. Even though three of the 168 seedlings were described as being positive by enzymelinked immunosorbent assay (ELISA) on the cotyledon and leaf tissues in the transplanting stage, none of the tomato plants developed any symptoms. Infectivity of PepMV in the three alleged ELISApositive plants was also not validated with back-inoculation. Thus, the true rate of seed transmission from tomato seed, if any, remains in question. These conflicting results have caused confusion in seed health tests and in seed treatment. It is therefore important to evaluate the potential seed transmission using large-scale seedling grow-out assays with highly PepMV-contaminated commercial tomato seeds.

The objectives of the present study were to evaluate the potential seed transmission through large-scale seedling grow-out, to investigate the efficiency of PepMV transmission through mechanical inoculation with tomato seed, and to determine the exact location of PepMV on tomato seed, floral parts, and fruit tissues. In addition, the potential seed transmission of PepMV in Nicotiana benthamiana was evaluated.

\section{MATERIALS AND METHODS}

Seed source and virus culture. The seed sample used in this study was a gift from an anonymous seed company. This commercial tomato seed lot (cultivar unknown) was originally produced in Chile in 2003. A routine seed health assay with ELISA showed that this seed lot contained PepMV (all 12 subsamples of 250 seeds each was positive with a mean absorbance value of $1.271 \pm 0.150$ ) and was free from two other potential seedborne viruses in tomato (Tomato mosaic virus [ToMV] and Tobacco mosaic virus [TMV]). A virus culture was obtained through mechanical inoculation of seed extract onto $N$. benthamiana. When inoculated on tomato plants (cv. Horizon), the isolated virus induced pepino mosaic-like symptoms (yellow mosaic, leaf blister) on tomato. It was subsequently determined that this virus culture actually contained two genetic variants of PepMV (Ch1 and Ch2), and their complete genome sequences have been determined (11).

ELISA for PepMV. A seed health assay for PepMV was performed following the standard method as recommended by the International Seed Health Initiative for Vegetables (ISHI_Veg) (published online). This standard method recommends conducting an ELISA of 3,000 seeds in 12 subsamples of 250 seeds. Seeds in each subsample were ground to fine powder with a Homex 6 homogenizer (BioReba AG, Reinach, Switzerland). Tissue extract was prepared by soaking the processed seed powder in 10 volumes of $1 \times$ extraction buffer (BioReba) on ice with occasional mixing by hand to facilitate the release of virus particles into the extraction buffer. The source of the antibody used to detect PepMV was Agdia (Elkhart, IN). Direct antibody sandwich ELISA (DASELISA) was conducted following the manufacturer's instructions. The absorbance value was taken $1 \mathrm{~h}$ after substrate hydrolysis at $\mathrm{OD}_{405 \mathrm{~nm}}$ in a Spectramax Plus384 (Molecular Devices, Sunnyvale, CA). A sample was considered positive if its absorbance value was at least twice that of the mean healthy control of PepMV-free tomato seeds. If ELISA detected no PepMV in the seed extract, the test was concluded. However, if ELISA detected the presence of PepMV, then bioassay on 
$N$. benthamiana should be performed to confirm virus infectivity.

Bioassay on tomato or $N$. benthamiana seedlings. To evaluate whether the virus on the PepMV-contaminated commercial tomato seed could serve as inoculum to initiate virus infection, mechanical inoculation experiments were set up to determine the efficiency of PepMV infection on tomato (cv. Horizon) or $N$. benthamiana seedlings. To prepare for the bioassay, seed samples were ground to a fine powder with mortar and pestle or using a Polytron PT10-35 homogenizer (Kinematica AG, Lucerne, Switzerland) in (1:10 wt/vol) ice-cold phosphate buffered saline $\left(137 \mathrm{mM} \mathrm{NaCl}, 8 \mathrm{mM} \mathrm{Na} \mathrm{HPO}_{4}\right.$, $1.5 \mathrm{mM} \mathrm{KH} \mathrm{KO}_{4}, 2.7 \mathrm{mM} \mathrm{KCl}$, and 7.9 $\mathrm{mM} \mathrm{Na} 2 \mathrm{SO}_{3}, \mathrm{pH}$ 7.0). To prevent contamination between subsamples, the polytron probe was disinfected with triple rinses, first in water, followed by $70 \%$ ethanol, and then water again before use for the next sample. Mechanical inoculation was done with gentle rubbing on lightly Carborundum-dusted (320 mesh grit powder, Fisher Scientific, Pittsburgh, PA) leaves of $N$. benthamiana (three to five leaf stage) with a cotton swab soaked in the prepared seed extract. After washing off excess Carborundum, the inoculated plants were placed under shade for several hours to protect them from direct sunlight damage to the inoculated leaves. Gloves were worn during inoculation and changed between samples to prevent any accidental cross contamination between samples. Care was taken to maintain space between the tested plants, especially immediately following inoculation. The inoculated plants were subsequently transferred to a greenhouse and maintained at 20 to $30^{\circ} \mathrm{C}$ with 12 to 14 $\mathrm{h}$ natural lighting for the duration of the test. The final symptom reading was conducted 4 weeks postinoculation, and systemic leaf tissues from each individual plant were collected to confirm the presence of PepMV by ELISA.

Seedling grow-out assay. To identify any potential natural seed transmission of PepMV in tomato, the highly PepMVcontaminated commercial hybrid tomato seed lot described above was used. In two separate experiments, a total of 10,000 tomato seeds were evaluated for seedling grow-out. For each experiment, an estimated 5,000 tomato seeds (as determined by weight) were divided into 10 subsamples (500 seeds each) and planted on 10 plastic flat planting trays $(28 \times 54 \mathrm{~cm})$ in commercial potting soil (Metro-Mix 360, Sun Gro, Bellevue, WA). Seedlings were maintained in a greenhouse $\left(20\right.$ to $30^{\circ} \mathrm{C}$, 12 to $14 \mathrm{~h}$ natural light) for symptom expression. Care was taken to avoid or minimize any unnecessary physical contact to the germinating seedlings during maintenance, watering, and symptom observation, although natural contacts among seedlings were permitted. Plants were scored for the presence or absence of symptoms 4 weeks after seedling germination. ELISA was used to confirm any latent virus infection with composite leaf tissue samples. Leaf tissues were collected from each seedling and bulked into 20 composite samples (250 seedlings per sample) and processed in 1:10 (wt/vol) tissue extraction buffer (BioReba AG) with Homex-6 homogenizer. Samples were tested in duplicate wells per sample. To eliminate any potential escape during the first ELISA, a second symptom reading and ELISA were conducted 1 week thereafter (total of 5 weeks postgermination) with newly collected tissue samples. To confirm that the ELISA-negative samples contained no viable virus particles, bioassay was conducted with a new tissue extract from each composite sample by inoculation onto $N$. benthamiana plants.

Immunocapture real-time reverse transcription polymerase chain reaction (RT-PCR). To determine the exact location where PepMV is likely accumulated in different organ tissues from a PepMVinfected tomato plant, immunocapture real-time RT-PCR as described by Ling et al. (12) was used to analyze the presence of PepMV in tissue samples including seed parts (seed coat and embryos from immature or mature seeds), floral organs (stigma, sepal, petal, anther, and anther filament) or fruit tissues (seed membrane and fruit pulp). Immunocapture sample preparation for each tomato tissue was performed by incubating the tissue extract in PepMV antibody-coated PCR microtubes. The real-time RT-PCR for PepMV detection was performed using the same conditions as determined previously (12). Experiments were repeated at least twice for each tissue type.

Location of PepMV on mature tomato seed. For each experiment, a total of 3,000 tomato seeds were divided into 10 subsamples of approximately 300 seeds each. In order to physically separate seed coat from embryo, PepMVcontaminated tomato seeds were pregerminated in petri dishes on moist tissue paper at $25^{\circ} \mathrm{C}$ for 5 to 7 days. The seed coat (testa) and embryo (including cotyledons and root tips) from each germinating seedling in a subsample were carefully separated with a pair of fine-point forceps and placed into respectively labeled clean tubes. The separated seed coat or embryo samples were soaked in $10 \% \mathrm{Na}_{3} \mathrm{PO}_{4}$ briefly and triple-washed with sterile distilled water to remove any remainders of tissue or potential surfacelocating virus particles. To prevent any potential contamination between samples, tools (forceps) were cleaned by soaking them in a $70 \%$ ethanol solution and wiping clean before use. The resulting 10 subsamples of seed coats or embryos, respectively, were processed in the icecold phosphate buffered saline for
ELISA, real-time RT-PCR, and bioassay on $N$. benthamiana plants.

Location of PepMV on seed parts, floral organs, and fruits. To investigate the virus distribution pattern in various tissues, floral organs including stigma, sepal, petal, anther, and anther filament from 6 to 10 flowers in a PepMV-infected tomato plant (cv. Horizon) were carefully and cleanly dissected and bulked into each individual sample for ELISA. Immature seeds were extracted from green tomato fruits produced on the PepMV-infected tomato plants. Seed coats and embryos from freshly extracted seeds were carefully separated. Several tissue types, including embryo, seed coat, seed membrane, and fruit pulp, were collected individually. To avoid cross contamination from fruit juice, each isolated seed part (embryo, seed coat, and seed membrane) was triple-washed with sterile distilled water prior to use for tests, including ELISA, real-time RT-PCR, and back-inoculation.

Evaluating $N$. benthamiana seeds for virus transmission. Both ELISA and seedling grow-out were used for this evaluation. Fresh seeds harvested from seed pods of a PepMV-infected plant were triple-washed with sterile distilled water. Seeds harvested from a healthy N. benthamiana plant were used for controls. In ELISA, bulked samples consisting of 100 $N$. benthamiana seeds were ground to fine powder with a mortar and pestle in tissue extraction buffer. Pods and leaf tissues collected from the same PepMV-infected $N$. benthamiana plant were also included as positive controls. For seedling grow-out, approximately 1,000 seeds collected from the PepMV-infected $N$. benthamiana plant were planted in the same way as described above for tomato seed. ELISA was used to rule out any potential latent PepMV infection on the germinating seedlings.

Seed germination. Seed germination was conducted in house or outsourced to a commercial seed testing laboratory (e.g., STA Laboratories, Gilroy, CA). For each germination test, a total of 400 seeds per sample (100 seeds per subsample) were placed between moist filter papers and incubated in a growth chamber $\left(25^{\circ} \mathrm{C}, 16 \mathrm{~h}\right.$ light period) for the duration of a test (14 days). Two readings, first at 7 days and second at 14 days, were recorded.

\section{RESULTS}

Seed transmission of PepMV was not observed in two large-scale seedling grow-out assays. The germination rate for this commercial tomato seed lot was previously determined to be around $98 \%$. Using these highly PepMV-contaminated tomato seeds, all 12 subsamples of 250 seeds were positive by ELISA with a mean absorbance at $1.271 \pm 0.150$. A total of 10,000 seedlings were evaluated in two separate seedling grow-out tests $(5,000$ seedlings each). None of the 10,000 seed- 
lings in two separate experiments developed any sign of PepMV infection. Nevertheless, ELISA was performed to determine whether these germinated seedlings were indeed PepMV-free. In the first ELISA with 20 bulked samples of 250 seedlings each, all samples were negative, with ELISA absorbance values ranging from 0.046 to 0.105 with a mean of 0.071 . In this test, the healthy cutoff was set at 0.148 , two times that of the mean healthy control. The second symptom reading, conducted 1 week thereafter, again confirmed no virus infection status on all germinated seedlings. A new ELISA with freshly collected tissue samples was again negative $\left(\mathrm{OD}_{405 \mathrm{~nm}}: 0.085\right.$ to 0.139 with a healthy cutoff at 0.198$)$. N. benthamiana plants back-inoculated with tissue extract prepared from the germinating seedlings showed no virus infection. These negative results in the seedling grow-out, in ELISA, and in back-inoculation suggested that infectious PepMV particles were not present in seedling leaf tissues. Although PepMV was seedborne, it was not seedtransmitted in tomato under greenhouse growing conditions.

Efficient mechanical transmission of PepMV from tomato seed to tomato and $N$. benthamiana seedlings. Infectivity of PepMV on the tomato seed was determined with bioassay through inoculation of $N$. benthamiana plants. In this test, all $10 \mathrm{~N}$. benthamiana seedlings inoculated with the inoculum prepared from seed extract of 250 tomato seeds developed typical PepMV infection. Using the same seed extract as inoculum, only two of 40 inoculated tomato plants exhibited virus infection (5\% infection rate). These results indicated that $N$. benthamiana was more easily infected with PepMV than was tomato. Therefore, $N$. benthamiana was used as an indicator plant to test for PepMV infection.

PepMV was located in seed coat but not in embryo of tomato seeds. Ability and efficiency of virus transmission from tomato seeds to seedlings are influenced by the location of PepMV particles on the tomato seed. However, the exact location of PepMV on tomato seed parts had not been determined. Results from initial experiments in ELISA and bioassays showed that under normal germination conditions, PepMV was seedborne but not seedtransmitted to seedlings. However, the virus could be effectively transmitted to tomato seedlings by mechanical means. Evaluating where the virus was actually located on tomato seed may help to explain why PepMV possessed such unusual properties in virus transmission. The location of PepMV in tomato seed was determined by serological test, by real-time RTPCR, and by infectivity assay. When ELISA was performed on samples with separated seed coats or embryos, positive reactions were observed only from the seed coat subsamples $\left(\mathrm{OD}_{405 \mathrm{~nm}}\right.$ ranging 0.230 to 4.000 ), while all embryo subsamples were negative $\left(\mathrm{OD}_{405 \mathrm{~nm}}\right.$ ranging 0.051 to 0.106 , the threshold for positive was 0.150). In real-time RT-PCR, the seed coat tissue had a positive reaction $\left(\mathrm{C}_{\mathrm{T}}: 25.57\right)$, whereas a negative result $\left(\mathrm{No}_{\mathrm{T}}\right)$ was detected in the embryo tissues isolated from the PepMV-infested tomato seeds. In addition to the positive reactions in ELISA and real-time RT-PCR, virus infectivity in seed coat samples was also demonstrated on $N$. benthamiana plants by bioassay. The localization of PepMV on the tomato seed coat rather than in the embryo likely explained the reason why PepMV was seedborne but not seed-transmitted in tomato seeds.

Distribution of PepMV in tomato seed parts, floral organs, and fruits of an infected tomato plant. To investigate further the pattern of PepMV distribution on immature seed and floral organs during plant development in a PepMV-infected tomato plant, techniques including realtime RT-PCR, serological test, and infectivity assay were again used for evaluation. The results from ELISA showed that immature embryos were free from PepMV $\left(\mathrm{OD}_{405 \mathrm{~nm}}: 0.08 \pm 0.02\right)$. Although samples from fresh seed coat alone had a positive reaction, the absorbance value $\left(\mathrm{OD}_{405 \mathrm{~nm}}\right.$ : $0.25 \pm 0.01$ ) was much lower than that of the seed membrane $\left(\mathrm{OD}_{405 \mathrm{~nm}}: 1.04 \pm 0.40\right)$ or fruit pulp $\left(\mathrm{OD}_{405 \mathrm{~nm}}\right.$ : $\left.1.63 \pm 0.16\right)$ extracted from PepMV-infected tomato fruits (Table 1). Real-time RT-PCR also showed a negative reaction in the immature embryo $\left(\mathrm{C}_{\mathrm{T}}:\right.$ 31.02). Because the threshold $\left(\mathrm{C}_{\mathrm{T}}\right)$ of the mean healthy seed tissue was 32.84 in this test, the $\mathrm{C}_{\mathrm{T}}$ for a positive was arbitrarily set at 30.00 . However, positive reactions were shown in seed coat $\left(\mathrm{C}_{\mathrm{T}}\right.$ : 25.57), in membrane $\left(\mathrm{C}_{\mathrm{T}}: 18.49\right)$, and in fruit pulp $\left(\mathrm{C}_{\mathrm{T}}\right.$ : 17.84) (Table 1). Bioassays confirmed the infectivity of PepMV in the seed coat but not in immature embryos. Other maternal tissues such as seed membrane, fruit pulp, and other flower organs (including stigma, petal, anther, and anther filament) also contained infectious viruses (Table 1).

PepMV was neither seedborne nor seed-transmitted in $N$. benthamiana. In addition to the study of seedborne and seed transmissibility of PepMV in tomato, similar experiments were also conducted to investigate the seed transmissibility of PepMV in the experimental host $N$. benthamiana. Seeds harvested from PepMVinfected $N$. benthamiana plants were first evaluated with ELISA. A strong positive ELISA reaction was obtained from seed pods $\left(\mathrm{OD}_{405 \mathrm{~nm}}\right.$ : 3.46$)$. Even though preliminary ELISA showed a high absorbance reading $\left(\mathrm{OD}_{405 \mathrm{~nm}}: 1.90\right)$ with newly harvested seeds (regarded as dirty seed) collected directly from PepMV-infected $N$. benthamiana plants, surface-cleaned seeds (triple-water rinses) were actually negative in ELISA ( $\mathrm{OD}_{405 \mathrm{~nm}}$ : 0.04) as well as in Bioassay (data not shown). Furthermore, none of the germinating seedlings developed virus infection in a seedling grow-out test of over 1,000 seedlings based on symptom expression, and tested negative in ELISA (data not shown). These results showed that, unlike in tomato seeds where PepMV was seedborne but not seedtransmitted, PepMV in N. benthamiana was neither seedborne nor seedtransmitted. This nonseedborne nature of PepMV in $N$. benthamiana was similar to the nonseedborne nature of PepMV in its originally identified host plant, pepino (9).

\section{DISCUSSION}

This paper reports that PepMV is a seedborne, but not seed-transmitted virus on tomato. The mechanical transmission of

Table 1. Virus distribution in tomato seed parts, floral organs, and fruits in a Pepino mosaic virus (PepMV) infected tomato plant

\begin{tabular}{llcc}
\hline Tissue types & ELISA $^{\mathbf{x}}$ & Real-time RT-PCR $^{\mathbf{y}}$ & Bioassay $^{\mathbf{z}}$ \\
\hline Embryo & $0.08(-)$ & $35.40(-)$ & - \\
Seed coat & $0.25(+)$ & $29.84(+)$ & + \\
Seed membrane & $1.04(++)$ & $18.49(+)$ & + \\
Fruit pulp & $1.63(++)$ & $17.84(+)$ & + \\
Stigma & $2.03(+++)$ & $24.94(+)$ & + \\
Sepal & $1.81(++)$ & $16.71(+)$ & + \\
Petal & $1.91(++)$ & $16.54(+)$ & + \\
Anther & $2.04(+++)$ & $16.79(+)$ & + \\
Anther filament & $1.59(++)$ & $24.19(+)$ & + \\
Healthy check & $0.08(-)$ & $35.00-\mathrm{No} \mathrm{C}_{\mathrm{T}}$ & - \\
Threshold for positive & 0.16 & 35.00 & + \\
PepMV-leaf & $3.88(++++)$ & $16.04(+)$ & + \\
\hline
\end{tabular}

${ }^{\mathrm{x}}$ Enzyme-linked immunosorbent assay. Average absorbance values from duplicate wells of each sample were presented. $(-)$ : $<0.160 ;(+)$ : 0.161-1.000; (++): 1.001-2.000; $(+++): 2.001-3.000$; and $(++++): 3.001-4.000$. Threshold for a positive ELISA $\left(\mathrm{OD}_{405 \mathrm{~nm}}: 0.160\right)$ was twice that of the average health check.

y Immunocapture real-time reverse transcription-polymerase chain reaction (RT-PCR) was conducted as described (12), the threshold for positive (+) was arbitrarily set at $\mathrm{C}_{\mathrm{T}}=35.00$ based on the range of $\mathrm{C}_{\mathrm{T}}$ values on the healthy tissue sample controls.

${ }^{\mathrm{z}}$ Bioassay was conducted by mechanical inoculation of each tissue extract onto two Nicotiana benthamiana plants, which were observed for symptom expression under greenhouse conditions. -: without infection; +: with infection. 
PepMV from virus-containing seeds to seedlings is likely responsible for initiating a new infection on tomato. To my knowledge, this is the first report to determine the localization of PepMV in tomato seed coat but not in the embryo. The unusual seedborne nature of PepMV in tomato seed may be due to its hairy seed coat structure, because the same virus was not shown to be seedborne either in $N$. benthamiana (this study) or in pepino (9). Although efforts were made during the seed coat and embryo tissue separation step to clean out any remaining fruit pulp tissue through triple rinsing with sterile distilled water of the isolated seed coats, it is possible that some small pieces of fruit pulp or gelatinous seed membrane tissue may still stick to the seed coats. Nevertheless, this surface-locating nature of PepMV on tomato seeds makes it possible for seed treatment (3).

There have been some conflicting results regarding the seed-transmissibility of PepMV in tomato. With a small number of seeds, Salomone and Roggero (21) observed no seed transmissibility in seedling grow-out tests. A recent report by Cordoba-Selles et al. (3), however, reported $1.84 \%$ seed-to-seedling transmission of PepMV. In the present study using over 10,000 highly PepMV-contaminated commercial tomato seeds for seedling grow-out tests in a greenhouse, no seed to seedling transmission of the virus was detected. The conclusion of no seed to seedling transmission of PepMV was supported by the negative ELISA on the naturally germinating seedlings and no positive infection through back-inoculation to $N$. benthamiana plants. In the study conducted by Cordoba-Selles et al. (3), back-inoculation was not used to recover infectious PepMV from the $1.84 \%$ ELISA-positive seedlings. Furthermore, because authors in that study did not present the ELISA absorbance value on virus titers from the positive seedlings, direct comparison with the present study was not possible. The present study is in agreement with that of Salomone and Roggero (21) with the conclusion of no true seed to seedling transmission during normal seedling germination. However, the present study also showed that with the ease of mechanical transmission of PepMV from tomato seeds to seedlings, the virus contaminated seed could potentially serve as a source of initial virus inoculum.

It was estimated that approximately $18 \%$ of the described plant viruses are seed-transmitted in one or more hosts (8). Transmission of viruses through seed can be of considerable ecological significance for virus perpetuation, dissemination, and survival through years in seed storage. Virus perpetuation by infected seed is seemingly the perfect survival strategy since it serves as a protective link between crop-growing seasons. However, it is prudent to distinguish between seedborne viruses in general and viruses that depend upon seed-transmission for perpetuation. Depending on the situation, the rate of seed-transmission alone may not be a good indicator of epidemiological significance for the virus. Even though PepMV is not seed-transmitted, with the ease of mechanical transmission of the virus, any direct or indirect contact to a healthy tomato plant with a PepMV-contaminated tomato seed could potentially result in initiating a virus infection in a new facility or even in a new area. A new virus strain could also be introduced in this way to an area where PepMV is already endemic. Therefore, in commercial greenhouse tomato production, it is extremely critical to plant certified PepMV-free tomato seeds as the first preventive measure in disease management.

In order to make an accurate assessment in the significance of tomato seed in the epidemiology of PepMV in tomato, it is necessary to reiterate the terms seedborne and seed-transmission. Seedborne means the virus is carried on seeds (either as a contaminant or in the seed coat) but does not necessarily infect the seedlings germinated naturally from the virus-contaminated seeds. Seed-transmission means the virus (usually located in embryo) is capable of infecting the naturally germinating seedlings $(5,8,16)$. The presence of virus in seed, even in the embryo, does not always lead to seedling infection. One exception to this is in the case of TMV, where the virus is carried as a contaminant on the surface of tomato and pepper seeds that can infect seedlings during germination and early growth (16). It is likely that a seedborne virus may initiate the infection of the germinating seedling through wounds caused by the germination process or by mechanical means. In the case of PepMV, because a natural virus infection was not found in the germinating tomato seedlings, the initial virus infection may be initiated through mechanical means, including the handling of seedlings with dirty hands or tools that had come to contact with PepMV-containing seeds. Once a new virus infection is established, secondary transmission of PepMV could be facilitated from plant to plant through many of the hands-on activities in a greenhouse tomato production cycle.

Since PepMV-specific antibody can detect the presence of virus coat protein from either infectious or nonviable virus particles, a positive ELISA result can only provide an indication for the presence of PepMV on the seed. Bioassay with mechanical inoculation onto an indicator plant ( $N$. benthamiana) is a crucial step in a seed health assay to determine PepMV infectivity. This is especially important when treated seed samples are used. For a seedborne but not seed-transmitted virus like PepMV, an ELISA-positive seed sample does not necessarily mean that the seed is not safe to plant, especially if the seed had been treated previously. Nonviable virus particles in a treated seed sample could still produce a positive reading when tested in ELISA. If this is the case, a seed sample with a positive ELISA reading may actually pose no risk of virus transmission. Nevertheless, presence of a false-positive ELISA reading would be undesirable for the certification of virus-free seed. The true virus infectivity, however, can be determined with a bioassay through mechanical inoculation of seed extract on the indicator plant, such as $N$. benthamiana. The data presented in this study clearly support the notion that PepMV is a seedborne but not seed-transmitted virus. For such a virus, a seedling grow-out test would yield no infection on germinating seedlings. A false negative reading would, however, have a serious consequence in potentially introducing PepMV into a new area. Similar to the situation with TMV in tomato and pepper seed $(2,16,23)$, with its efficient mechanical transmission, a viruscontaminated tomato seed lot can still serve as a source of initial virus inoculum for PepMV. Whole-seed serological assays are therefore not suitable to estimate the rate of seed transmission because viral presence in the seed coat relates little to virus transmission from seeds to seedlings $(7,15)$. Therefore, it is extremely important to conduct a bioassay through indexing of the ELISA-positive seed samples onto $N$. benthamiana or even tomato seedlings. Presence of PepMV can be evaluated with symptom expression on inoculated $N$. benthamiana plants and confirmed with ELISA as suggested by ISHI_Veg for seed health test of tomato seed for PepMV.

\section{ACKNOWLEDGMENTS}

I thank Andrea Gilliard and Emily Walters for their excellent technical assistance and C. Shaker Kousik and Howard Harrison for critical reading of the manuscript.

\section{LITERATURE CITED}

1. Aguilar, J. M., Hernandez-Gallardo, M. D., Cenis, J. L., Lacasa, A., and Aranda, M. A. 2002. Complete sequence of the Pepino mosaic virus RNA genome. Arch. Virol. 147:2009-2015.

2. Broadbent, L. 1965. The epidemiology of tomato mosaic. XI. Seed transmission of TMV. Ann. Appl. Biol. 56:177-205.

3. Cordoba-Selles, M. C., Garcia-Randez, A., Alfaro-Fernandez, A., and Jorda-Gutierrez, C. 2007. Seed transmission of Pepino mosaic virus and efficacy of tomato seed disinfection treatments. Plant Dis. 91:1250-1254.

4. Cotillon, A. C., Girard, M., and Ducouret, S. 2002. Complete nucleotide sequence of the genomic RNA of a French isolate of Pepino mosaic virus (PepMV). Arch. Virol. 147:22312238.

5. De Assis Filho, F. M., and Sherwood, J. L. 2000. Evaluation of seed transmission of Turnip yellow mosaic virus and Tobacco mosaic virus in Arabidopsis thaliana. Phytopathology 90:1233-1238.

6. French, C. J., Bouthillier, M., Bernardy, M., Ferguson, G., Sabourin, M., Johnson, R. C., Masters, C., Godkin, S., and Mumford, R. 
2001. First report of Pepino mosaic virus in Canada and the United States. Plant Dis. 85:1121.

7. Gillaspie, A. G., Jr., Hopkins, M. S., and Pinnow, D. L. 1993. Relationship of cowpea seedpart infection and seed transmission of blackeye cowpea mosaic potyvirus in cowpea. Plant Dis. 77:875-877.

8. Johansen, E., Edwards, M. C., and Hampton, R. O. 1994. Seed transmission of viruses: Current perspectives. Annu. Rev. Phytopathol. 32:363-386.

9. Jones, R. A. C., Koenig, R., and Lesemann, D. E. 1980. Pepino mosaic virus, a new potexvirus from pepino Solanum muricatum. Ann. Appl. Biol. 94:61-68.

10. Jorda, C., Lazaro-Perez, A., MartinezCulebras, P., Abad, P., Lacasa, A., and Guerrero, M. M. 2001. First report of Pepino mosaic virus on tomato in Spain. Plant Dis. 85:1292.

11. Ling, K. S. 2007. Molecular characterization of two Pepino mosaic virus variants from imported tomato seed reveals high levels of sequence identity between Chilean and US isolates. Virus Genes 34:1-8.

12. Ling, K. S., Wechter, W. P., and Jordan, R. 2007. Development of a one-step immunocapture Real-time TaqMan RT-PCR assay for the broad spectrum detection of Pepino mosaic virus. J. Virol. Methods 144:65-72.

13. Lopez, C., Soler, S., and Nuez, F. 2005. Comparison of the complete sequences of three dif- ferent isolates of Pepino mosaic virus: Size variability of the TGBp3 protein between tomato and L. peruvianum isolates. Arch. Virol. 150:619-627.

14. Maroon-Lango, C. J., Guaragna, M. A., Jordan, R. L., Hammond, J., Bandla, M., and Marquardt, S. K. 2005. Two unique US isolates of Pepino mosaic virus from a limited source of pooled tomato tissue are distinct from a third (European-like) US isolate. Arch. Virol. 150:1187-1201.

15. Maury, Y., Bossennec, J. M., Boudazin, G., Hampton, R., Pietersen, G., and Maguire, J. 1987. Factors influencing ELISA evaluation of transmission of Pea seed-borne mosaic virus in infected pea seed: Seed group size and seed decortication. Agronomie 7:225-230.

16. Mink, G. I. 1993. Pollen- and seed-transmitted viruses and viroids. Annu. Rev. Phytopathol. 31:375-402.

17. Mumford, R. A., and Metcalfe, E. J. 2001. The partial sequencing of the genomic RNA of a UK isolate of Pepino mosaic virus and the comparison of the coat protein sequence with other isolates from Europe and Peru. Arch. Virol. 146:2455-2460.

18. Pagan, I., Cordoba-Selles, M. C., MartinezPriego, L., Fraile, A., Malpica, J. M., Jorda, C., and Garcia-Arenal, F. 2006. Genetic structure of the population of Pepino mosaic virus infecting tomato crops in Spain. Phytopathology 96:274-279.

19. Pospieszny, H., and Borodynko, N. 2006. New
Polish isolate of Pepino mosaic virus highly distinct from European tomato, Peruvian, and US2 strains. Plant Dis. 90:1106.

20. Roggero, P., Masenga, V., Lenzi, R., Coghe, F., Ena, S., and Winter, S. 2001. First report of Pepino mosaic virus in tomato in Italy. Plant Pathol. 50:798.

21. Salomone, A., and Roggero, P. 2002. Host range, seed transmission and detection by ELISA and lateral flow of an Italian isolate of Pepino mosaic virus. J. Plant Pathol. 84:65-68.

22. Soler, S., Prohens, J., Diez, M. J., and Nuez, F. 2002. Natural occurrence of Pepino mosaic virus in Lycopersicon species in Central and Southern Peru. J. Phytopathol. 150:49-53.

23. Taylor, R. H., Grogan, R. G., and Kimble, K. A. 1961. Transmission of Tobacco mosaic virus in tomato seed. Phytopathology 51:837842 .

24. van der Vlugt, R. A. A., Stijger, C. C. M. M. Verhoeven, J. Th. J., and Lesemann, D.-E. 2000. First report of Pepino mosaic virus on tomato. Plant Dis. 84:103.

25. Verhoeven, J. Th. J., van der Vlugt, R. A. A. and Roenhorst, J. W. 2003. High similarity between tomato isolates of Pepino mosaic virus suggests a common origin. Eur. J. Plant Pathol. 109:419-425.

26. Zhang, Y., Shen, Z. J., Zhong, J., Lu, X. L., Cheng, G., and Li, R. D. 2003. Preliminary characterization of Pepino mosaic virus Shanghai isolate (PepMV-Sh) and its detection with ELISA. Acta Agric. Shanghai 19:90-92. 